\begin{tabular}{|} 
Ambiente \& Água - An Interdisciplinary Journal of Applied Science \\
ISSN 1980-993X - doi:10.4136/1980-993X \\
www.ambi-agua.net \\
E-mail: ambi.agua@gmail.com
\end{tabular}

\title{
Incêndios no Pantanal de Corumbá, MS: modelagem e previsão a partir das técnicas de análise multivariada
}

\author{
ARTICLES doi:10.4136/ambi-agua.2024
}

Received: 08 Oct. 2016; Accepted: 24 Jul. 2018

\author{
Hevelyne Henn da Gama Viganón ${ }^{1,2 *}$; Celso Correia de Souza ${ }^{1}$; \\ Marcia Ferreira Cristaldo ${ }^{1,3}$; José Francisco dos Reis Neto ${ }^{4}$; \\ Leandro de Jesus ${ }^{1,3}$
}
${ }^{1}$ Universidade para o Desenvolvimento do Estado e da Região do Pantanal (UNIDERP), Campo Grande, MS, Brasil Pós-Graduação em Meio Ambiente e Desenvolvimento Regional. E-mail: hevelyne.vigano@ifms.edu.br, csouza939@gmail.com, marcia.cristaldo@ifms.edu.br, leandro.jesus@ifms.edu.br
${ }^{2}$ Instituto Federal de Mato Grosso do Sul (IFMS), Campo Grande, MS, Brasil Departamento de Matemática e Estatística. E-mail: hevelyne.vigano@ifms.edu.br ${ }^{3}$ Instituto Federal de Mato Grosso do Sul (IFMS), Campo Grande, MS, Brasil
Departamento de Informática e Robótica.E-mail: marcia.cristaldo@ifms.edu.br, leandro.jesus@ifms.edu.br ${ }^{4}$ Universidade para o Desenvolvimento do Estado e da Região do Pantanal (UNIDERP), Campo Grande, MS, Brasil Pós-Graduação em Produção e Gestão Agroindustrial (UNIDERP). E-mail: jfreisneto@gmail.com
${ }^{*}$ Corresponding author

\section{RESUMO}

Os incêndios e as queimadas que ocorrem no Pantanal causam grandes prejuízos à fauna e flora locais. A previsão de eventos é de grande importância por possibilitar que as catástrofes nesse ecossistema sejam amenizadas ou, até mesmo evitadas. Este estudo teve como objetivo avaliar as ocorrências de queimadas e incêndios no Pantanal Sul-Mato-Grossense, associadas às variáveis meteorológicas e realizar uma modelagem de previsão a partir de técnicas de análise multivariada de dados. As variáveis ambientais envolvidas nesse processo foram extraídas da base de dados do Centro de Previsão de Tempo e Estudos Climáticos do Instituto Nacional de Pesquisas Espaciais (INPE) e do banco de dados meteorológicos para ensino e pesquisa do Instituto Nacional de Meteorologia (INMET). Foram observadas que a temperatura, umidade relativa e radiação solar, possuem um relacionamento estreito com a ocorrência dos focos e as correlações resultantes foram satisfatórias para a aplicação das modelagens de previsão. A técnica de Regressão Linear Múltipla apresentou $41 \%$ de ajustamento e a técnica de Análise Auto-regressiva Integrada de Médias Móveis apresentou ajustamento de 66,5\% e desempenho geral de 68,4\%, tornando-a a metodologia mais recomendada para a previsão.

Palavras-chave: bioma pantaneiro, perfil climatológico, previsão de fogo.

\section{Fires in the Pantanal: modeling and forecasting using multivariate analysis techniques}

\section{ABSTRACT}

The occurrence of fires in Pantanal causes great damage to the local fauna and flora. Predicting these events is of great importance, enabling catastrophes in this ecosystem to be mitigated or even avoided. This study evaluated the occurrence of fires in Southern Pantanal 
associated with meteorological variables and created a predictive model using multivariate data analysis techniques. The environmental variables involved in this process were extracted from the database of the Center for Weather Forecasting and Climatic Studies of the National Institute of Space Research (INPE) and the meteorological database for teaching and research of the National Institute of Meteorology (INMET). It was observed that temperature, relative humidity and solar radiation have a close relationship with the occurrence of fires and the resulting correlations were considered satisfactory for the application of forecasting models. The Multiple Linear Regression technique presented an adjustment of $41 \%$ and the Integrated Averaging Analysis of Moving Averages presented an adjustment of $66.5 \%$ and a general performance of $68.4 \%$, making it the most-recommended forecasting methodology.

Keywords: biome pantaneiro, climatological profile, fire forecast.

\section{INTRODUÇÃO}

A ocorrência de incêndios no Pantanal Sul-Mato-Grossense representa uma ameaça para a conservação da biodiversidade local, com influência nas condições climáticas desse importante bioma, causando sérias consequências à manutenção de processos ecológicos, com reflexos inclusive na permanência do homem nesse habitat natural (Nogueira e Santos, 2015).

Em grande parte as queimadas e incêndios ocorrem por causas antrópicas (Trejo, 2008; Santos e Nogueira, 2015; White e White, 2016; Clemente et al., 2017). Na estação de estiagem encontram condições propícias a se propagarem em alta magnitude pois, os fatores climáticos e meteorológicos, tais como elevadas temperaturas, radiação solar, baixa umidade relativa do ar e vento, potencializam a sua ocorrência, visto que, o ar mais seco aumenta a evapotranspiração dos vegetais e favorece maior atuação da radiação solar sobre a superfície terrestre que, por sua vez, eleva a temperatura do ar, criando um ambiente facilmente propício ao processo de combustão (Deppe et al., 2004; Magi et al., 2012; Gonçalves e Vieira, 2013; Almeida et al., 2016).

Os danos decorrentes dos processos de queimadas e incêndios nos diversos componentes do bioma são: i) na vegetação ocorrem perdas de espécies nativas; ii) no solo ocorrem a destruição da camada orgânica, a sua exposição e o enfraquecimento; mudanças nas propriedades físicas (porosidade e penetrabilidade da água); deslizamentos e erosão; iii) na fauna ocorrem mortes dos animais, destruição de ninhos e modificação do habitat (animais migram por busca de alimentos e abrigo); iv) nas propriedades ocorrem destruição de casas, construções, veículos, maquinários e equipamentos diversos; v) na vida humana ocorrem problemas respiratórios decorrentes da poluição atmosférica , acidentes em estradas causados pela fumaça, e morte de pessoas envolvidas no combate ao fogo (Freitas et al., 2005; Pereira et al., 2012; Soares et al., 2009; Silva, 2014; Nunes et al., 2015).

A concentração de queimadas e incêndios ocorre entre os meses de julho a novembro, no bioma pantaneiro do Estado de Mato Grosso do Sul, também denominado de período JASON, caracterizado pela estação seca, onde os elementos climáticos contribuem fortemente com o processo de combustão da vegetação, registrando maior incidência no município de Corumbá (Viganó et al., 2017).

Estudos sobre a composição elementar das partículas de aerossol resultantes dessas queimadas mostram que a emissão de carbono grafítico (Black-carbon) durante a estação seca associada a elementos conhecidos como traçadores de emissões de queimadas, Enxofre (S), Potássio (K), Cloro (Cl), Cálcio (Ca) e Zinco (Zn), potencializam a ocorrência das queimadas e consequentemente o efeito estufa (Nogueira e Santos, 2015; Artaxo et al., 2006).

Diante da constatação dos grandes prejuízos causados pelo processo de combustão no bioma pantaneiro, a previsão, o controle e a fiscalização desses eventos são de considerável 
interesse às autoridades competentes e ao homem pantaneiro. E nessa perspectiva, determinar e prever a localização dos focos, os riscos e as ocorrências, representa um desafio para a elaboração de planos de conservação ou de manejo.

Segundo Silva (2014) e Morrison (2014), o meio mais eficiente e de baixo custo em países de grande extensão territorial, como o Brasil, é o monitoramento de queimadas e incêndios por meio de imagens orbitais de satélites pelo processo de sensoriamento remoto, que permite detectar e localizar os focos em tempo real. Outras ferramentas que podem ser utilizadas para a previsão de focos de queimadas são as técnicas de análise multivariada de séries temporais, através das quais, a partir de uma massa de dados sobre os valores de algumas variáveis meteorológicas que influenciam a ocorrência de queimadas e do seu número de focos, é possível obter uma previsão de ocorrência do fenômeno.

Desse modo, o objetivo deste trabalho foi utilizar conceitos de análise de regressão linear múltipla e de médias móveis autorregressivas integradas para determinar uma modelagem matemática de previsão sobre o número de focos de incêndios no bioma do Pantanal de Corumbá, levando-se em conta os níveis de medidas de uma série de variáveis meteorológicas, como preditoras e avaliar a correlação existente entre a ocorrência desses focos com essas variáveis meteorológicas.

\section{MATERIAIS E MÉTODOS}

\section{1. Área de Estudo}

Localizada na bacia do Alto Paraguai, na região Centro-Oeste do Brasil, às margens do rio Paraguai e na fronteira com a Bolívia, Corumbá é a quarta maior cidade do Estado de Mato Grosso do Sul, com cerca de 104.000 habitantes, a primeira cidade do Estado em extensão territorial, com aproximadamente $65.000 \mathrm{~km}^{2}$, onde $95 \%$ do total de sua área é bioma Pantanal (IBGE, 2016). Dentre os nove municípios que constituem o Pantanal Sul-Mato-Grossense, Corumbá representa $69 \%$ do bioma.

A localização geográfica de Corumbá está compreendida entre as coordenadas de 1900’32' ' S e 57³9'10' ' W e altitude de 118 m acima do nível do mar. A classificação climática de Corumbá, pertence ao tipo Aw (Clima Tropical Úmido Megatérmico), ou seja, clima Tropical de Altitude, com verões quentes e chuvosos, e inverno seco (Brasil, 1982).

De acordo com a Secretaria do Estado de Meio Ambiente e Desenvolvimento Econômico de Mato Grosso do Sul (SEMADE), e com a caracterização da cidade no RADAMBRASIL, o município de Corumbá divide-se em três regiões geomorfológicas: Região do Pantanal MatoGrossense; Região da Depressão do Alto Paraguai e Região da Bodoquena e Morrarias do Urucum-Amolar.

A cobertura vegetal predominante é o Cerrado típico do Pantanal, Cerrado Parque e Cerrado Arbóreo Aberto. Nos locais mais elevados, há áreas de matas e, ao sul do município, ocorre vegetação chaquenha; nas áreas mais baixas, encontram-se as palmeiras carandá e o paratudal, que trata de formação savânica alagável (Brasil, 1982; IBGE, 2016).

\subsection{Coleta de dados}

As informações das variáveis climatológicas e meteorológicas analisadas foram extraídas do (1) sistema de informações geográficas do banco de dados meteorológicos para ensino e pesquisa do Instituto Nacional de Meteorologia (INMET), utilizando a estação de coleta 83552 (1900'36' 'S e 57³8'60', W), e (2) do banco de dados da plataforma de coleta 31949 (1901'19.2'’ S e 57³9'7.2', W), disponível no Centro de Previsão de Tempo e Estudos Climáticos do Instituto Nacional de Pesquisas Espaciais (CPTEC- INPE) numa série temporal de 2005 a 2015.

Foram utilizadas 24 medidas diárias das variáveis preditoras (disponíveis de hora em hora

\section{IPABH}

Rev. Ambient. Água vol. 13 n. 5, e2024 - Taubaté 2018 
das 00h00 às 24h00) de cada variável meteorológica (total de 18 diferentes variáveis testadas), constituindo uma massa de dados inicial de 1.710.720 registros para toda a série temporal de janeiro a dezembro de 2005 a 2015. Para o período de julho a novembro (JASON), utilizaramse 712.800 registros na mesma série de medidas meteorológicas que foram tratadas para as modelagens. A base de dados da contagem dos focos, foi constituída apenas pelo total diário de focos detectados nos horários da passagem do satélite de referência, o que significa que essa contagem corresponde apenas à uma fração do total dos focos ocorridos, por este estudo se tratar da aplicação de técnicas de análise multivariada. Para a análise das variáveis meteorológicas do município, foram ainda calculados valores médios diários (série de 11 anos de dados), que foram calculadas a partir das médias compensadas diárias de cada variável, dada por $\mathrm{X}=\left(2 . \mathrm{X}_{00}+\mathrm{X}_{12}+\mathrm{X}_{\text {máx }}+\mathrm{X}_{\text {mín }}\right)$, onde $\mathrm{X}$ representa a variável em análise; $\mathrm{x}_{00}$ e $\mathrm{X}_{12}$ são os valores da variável em análise observadas à $00 \mathrm{~h} 00$ e às $12 \mathrm{~h} 00$ do tempo médio de Greenwich (TMG) e; os valores extremos $x_{\text {máx }}$ e $x_{\text {mín }}$ são os valores dos registros máximo e mínimo dentro do intervalo de $24 \mathrm{~h} 00$ de cada variável.

Com isso, a base de dados já tratada com as descrições acima, foi configurada com os dados médios compensados diários das variáveis meteorológicas (variáveis independentes) e com os quantitativos totais de referência diários dos focos (variável dependente), ou seja, cerca de 1650 dias avaliados.

Os dados da variável ambiental, número de focos, foram obtidos da Divisão de Geração de Imagens (DGI) do Instituto Nacional de Pesquisas Espaciais (INPE), que recebe e processa as imagens dos satélites de referência da série National Oceanic Atmospheric Administration NOAA-12 (de 2005 a 2007) e da National Aeronautics and Space Administration - NASA AQUA M-T (de 2008 a 2015), por meio, respectivamente, dos sensores Advanced Very High Resolution Radiometer - AVHRR e Moderate Resolution Imaging Spectroradiometer MODIS, cujas informações mantêm o mesmo padrão de amostragem temporal e de detecção, possuem horário de passagem em um mesmo ponto da Terra estável ao longo dos anos e são considerados isentos de falsas detecções em decorrência de reflexos do Sol na superfície terrestre. Cabe salientar que foram utilizadas medidas diárias disponíveis dos dados dos focos detectados pelos satélites de referência para a aplicação dos métodos multivariados de previsão de séries temporais, por apresentarem menores coeficientes de variação.

As variáveis meteorológicas selecionadas para o estudo da análise de correlação com o número de Focos $(\mathrm{N})$ foram: Temperatura Instantânea do $\operatorname{Ar}(\mathrm{oC})\left(\mathrm{T}_{\mathrm{Ar}}\right)$;Temperatura Máxima do $\operatorname{Ar}(\mathrm{oC})$ ( $\mathrm{T}_{\mathrm{Max}}$ ); Temperatura Mínima do $\operatorname{Ar}(\mathrm{oC})$ ( $\mathrm{T}_{\mathrm{Min}}$ ); Temperatura do Solo (oC) a

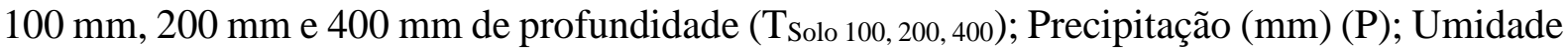
Relativa (\%) (URel); Umidade Absoluta (\%) (U $\left.\mathrm{U}_{\mathrm{Abs}}\right)$; Velocidade do Vento (m/s) a 10 metros (V Vento); Direção do Vento Máximo (oNV) (DMax); Direção do Vento (oNV) (D); Pressão Atmosférica (mB) ( $\left.\mathrm{P}_{\text {Atm }}\right)$; Radiação Solar Acumulada (MJ m-3) (Rsolar); Contagem de Água no Solo (m3) 100 mm, 200 mm e 400 mm de profundidade (Csolo 100, 200, 400).

\subsection{Técnicas de Análise Multivariadas para as previsões}

Para as modelagens das previsões dos focos foram utilizadas as técnicas de Regressão Linear Múltipla (RLM) e Análise Auto-Regressiva Integrada de Médias Móveis (ARIMA) (Hair Jr. et al., 2005; Morettin e Toloi, 2006; Caúla et al., 2016). Em ambos os modelos foram consideradas como variável dependente o número de focos e as variáveis meteorológicas como independentes. Os modelos foram testados com a série total de dados de janeiro de 2005 a dezembro de 2015 e, posteriormente, para o período de seca nos meses de julho a novembro com os dados das médias compensadas diárias das variáveis meteorológicas e as quantidades totais de referência de focos diários.

As técnicas foram executadas no software IBM-SPSS, sendo que, para o modelo RLM foi utilizada a configuração "stepwise”, com nível de significância de 5\%, e para o modelo ARIMA 
foi adotada a configuração "expert modeler”, com a previsão após o último caso em análise e até cinco passos adiante.

\subsection{Análises Estatísticas}

As análises estatísticas aplicadas aos modelos para os ajustes, a validação e o desempenho foram: a) Correlação de Pearson das variáveis independentes com relação a variável dependente; b) Análise de Variância (ANOVA) e estatística F; c) Significância (p-valor); d) R² e $\mathrm{R}^{2}$ ajustado; e) Teste de Durbin-Watson; f) Análise do Fator de Inflação da Variância; g) Análise dos Resíduos; h) Critério de Informação Bayesiano (BIC); i) Análise das funções de Autocorrelação dos Resíduos; j) Análise de Erros: raiz do erro quadrático médio (RMSE), erro percentual absoluto médio (MAPE) e erro absoluto médio (MAE) (Brito et al., 2016).

\section{RESULTADOS E DISCUSSÃO}

Foram detectados 27.902 focos nos meses de julho a novembro, no período de 2005 a 2015.A Tabela 1 apresenta a distribuição mensal dos focos, as médias mensais, os desvios padrão e os coeficientes de variação correspondentes, para a série analisada, ao longo dos meses de janeiro a dezembro, no período de 2005 a 2015, obtida de todos os sensores dos satélites que operam na faixa termal de $4 \mu \mathrm{m}$, sem sobreposição na leitura dos focos, para a varredura de 24 medidas horárias ao dia.

Tabela 1. Estatística mensal dos focos para os anos de 2005 a 2015, no bioma pantaneiro de Corumbá (MS).

\begin{tabular}{lccccccccccccc}
\hline & \multicolumn{10}{c}{ MÊS } \\
\cline { 2 - 5 } & JAN & FEV & MAR & ABR & MAI & JUN & JUL & AGO & SET & OUT & NOV & DEZ \\
\hline $\mathbf{2 0 0 5}$ & 3 & 45 & 22 & 37 & 134 & 224 & 535 & 2475 & 819 & 247 & 47 & 20 \\
$\mathbf{2 0 0 6}$ & 4 & 19 & 17 & 6 & 34 & 7 & 65 & 262 & 387 & 58 & 40 & 0 \\
$\mathbf{2 0 0 7}$ & 2 & 7 & 4 & 15 & 14 & 38 & 64 & 811 & 1443 & 205 & 32 & 5 \\
$\mathbf{2 0 0 8}$ & 8 & 3 & 12 & 14 & 5 & 3 & 27 & 166 & 889 & 252 & 24 & 157 \\
$\mathbf{2 0 0 9}$ & 161 & 88 & 45 & 219 & 859 & 255 & 265 & 591 & 855 & 662 & 331 & 36 \\
$\mathbf{2 0 1 0}$ & 13 & 38 & 62 & 67 & 34 & 61 & 332 & 646 & 773 & 288 & 174 & 221 \\
$\mathbf{2 0 1 1}$ & 52 & 13 & 1 & 1 & 11 & 18 & 43 & 80 & 258 & 98 & 344 & 492 \\
$\mathbf{2 0 1 2}$ & 154 & 59 & 75 & 38 & 118 & 76 & 469 & 2709 & 1792 & 522 & 53 & 86 \\
$\mathbf{2 0 1 3}$ & 93 & 33 & 92 & 28 & 36 & 4 & 75 & 254 & 460 & 207 & 259 & 105 \\
$\mathbf{2 0 1 4}$ & 94 & 64 & 16 & 49 & 12 & 17 & 24 & 58 & 191 & 271 & 111 & 16 \\
$\mathbf{2 0 1 5}$ & 77 & 41 & 11 & 20 & 30 & 188 & 152 & 708 & 781 & 341 & 200 & 290 \\
\hline Média & 60 & 37 & 32 & 45 & 117 & 81 & 186 & 796 & 786 & 286 & 147 & 130 \\
DP* & 59 & 26 & 30 & 59 & 239 & 91 & 189 & 884 & 509 & 231 & 116 & 147 \\
CV* & 98 & 70 & 94 & 131 & 204 & 112 & 102 & 111 & 65 & 81 & 79 & 113 \\
\hline
\end{tabular}

*DP: Desvio padrão; CV: Coeficiente de Variação (\%).

Observa-se, na Tabela 1, que as médias mensais no período de 2005 a 2015 crescem muito a partir do mês de julho, com picos em agosto e setembro, atingindo os seus valores médios máximos (796 focos e 786 focos, respectivamente). Os desvios padrão e, consequentemente, os coeficientes de variação têm valores altos, indicando uma grande heterogeneidade dos dados, o que é compreensível, pois, no primeiro semestre, acontecem poucos focos ao passo que no segundo semestre, estação seca, eles aumentam consideravelmente.

Com isso, o período selecionado para a aplicação das técnicas de previsão RLM e ARIMA, restringiu-se aos meses de julho, agosto, setembro, outubro e novembro de cada ano, denominado de período JASON, que corresponde aos meses em análises por se tratar de ser o 
período com a maior concentração de ocorrência de focos. Para a análise das séries temporais, foram utilizados os focos detectados apenas pelos satélites de referência NOAA-12 e NASA AQUA M-T, por apresentarem órbita polar, o que gera dados que podem ser analisados estatisticamente para as mesmas regiões ao longo dos anos com erros minimizados e efeitos atmosféricos corrigidos.

Para a execução da análise de regressão foi aplicada a transformação logarítmica de base 10 sobre os valores da variável número de focos $(\log (\mathrm{N}))$, para estabilizar a variância devido à grande variabilidade desses dados, pois, as previsões devem se caracterizar pela acurácia de seus resultados, pela simplicidade dos métodos empregados e pela confiabilidade estatística dos modelos empregados para gerar previsões.

A Tabela 2 mostra os resultados da correlação linear entre a variável $\log (\mathrm{N})$ e as variáveis meteorológicas preditoras.

Tabela 2. Correlação linear de Pearson (r) entre a variável Log $(\mathrm{N})$ e as variáveis meteorológicas preditoras, no período de 2005 a 2015, no município de Corumbá (MS).

\begin{tabular}{lclc}
\hline Variáveis & $\mathrm{r}$ & Variáveis & $\mathrm{r}$ \\
\hline $\mathrm{T}_{\text {Max }}\left({ }^{\circ} \mathrm{C}\right)$ & 0,383 & $\mathrm{C}_{\text {Solo100 }}(\mathrm{m} 3)$ & $-0,041$ \\
$\mathrm{~T}_{\text {Ar }}\left({ }^{\circ} \mathrm{C}\right)$ & 0,092 & $\mathrm{C}_{\text {Solo200 }}(\mathrm{m} 3)$ & $-0,144$ \\
$\mathrm{~T}_{\text {Min }}\left({ }^{\circ} \mathrm{C}\right)$ & 0,104 & $\mathrm{C}_{\text {Solo400 }}(\mathrm{m} 3)$ & $-0,038$ \\
$\mathrm{R}_{\text {Solar }}(\mathrm{MJ} \mathrm{m}-2)$ & 0,310 & $\mathrm{D}_{\text {Max }}(\mathrm{oNV})$ & $-0,080$ \\
$\mathrm{~T}_{\text {Solo100 }}\left({ }^{\circ} \mathrm{C}\right)$ & 0,183 & $\mathrm{D}(\mathrm{oNV})$ & $-0,090$ \\
$\mathrm{~T}_{\text {Solo200 }}\left({ }^{\circ} \mathrm{C}\right)$ & 0,165 & $\mathrm{P}(\mathrm{mm})$ & $-0,085$ \\
$\mathrm{~T}_{\text {Solot00 }}\left({ }^{\circ} \mathrm{C}\right)$ & 0,013 & $\mathrm{P}_{\text {Atm }}(\mathrm{mB})$ & $-0,019$ \\
$\mathrm{~V}_{\text {Vento10 }}\left(\mathrm{m} \mathrm{s}^{-1}\right)$ & 0,114 & $\mathrm{U}_{\text {Rel }}(\%)$ & $-0,564$ \\
$\mathrm{~V}_{\text {VentoMax }}\left(\mathrm{m} \mathrm{s}^{-1}\right)$ & 0,067 & $\mathrm{U}_{\text {Inst }}(\%)$ & 0,037 \\
\hline
\end{tabular}

Os valores negativos apresentados pelas correlações de Pearson (r) significam que, quanto menores os valores da variável preditora, maiores as chances de ocorrências de queimadas. Entretanto, se o valor de $\mathrm{r}$ for positivo, quanto maiores forem os valores das variáveis preditoras, maiores serão as chances de ocorrências de focos.

As variáveis meteorológicas que mais contribuem para a ocorrência de focos, de acordo com a análise de correlação para a aplicação dos métodos de RLM e ARIMA, são temperatura máxima, umidade relativa e radiação solar, as quais apresentaram os maiores valores absolutos de r. As demais variáveis preditivas que foram desconsideradas no modelo de regressão explicam o fenômeno em menos de $2 \%$, por se tratarem de termos indiretos, já associados com os fatores preditivos e apresentarem multicolinearidade com as demais variáveis.

Comparando os dados da Tabela 1 com os dados de Torres et al. (2011), sobre um estudo de ocorrência de incêndios em Juiz de Fora (MG), as correlações de Pearson mais significativas que se apresentaram mais próximas do presente trabalho foram as variáveis meteorológicas Radiação solar ( $r=0,357)$, Temperatura máxima $(r=0,182)$ e Umidade relativa $(r=-0,467)$, diferindo-se na temperatura pelas diferenças geográficas e climatológicas das duas regiões comparadas. Esse resultado, confirma a escolha das variáveis meteorológicas para as técnicas de modelagens aplicadas.

A variabilidade mensal para o período JASON para a série temporal de 2005 a 2015 do número de focos, da temperatura máxima, da umidade relativa e da radiação solar são apresentadas na Figura 1. É possível observar no gráfico da Figura 1 que a série que representa o número de focos (representado pelas barras) está disposta mensalmente, com cada barra correspondendo aos meses do período JASON (de julho a novembro). 


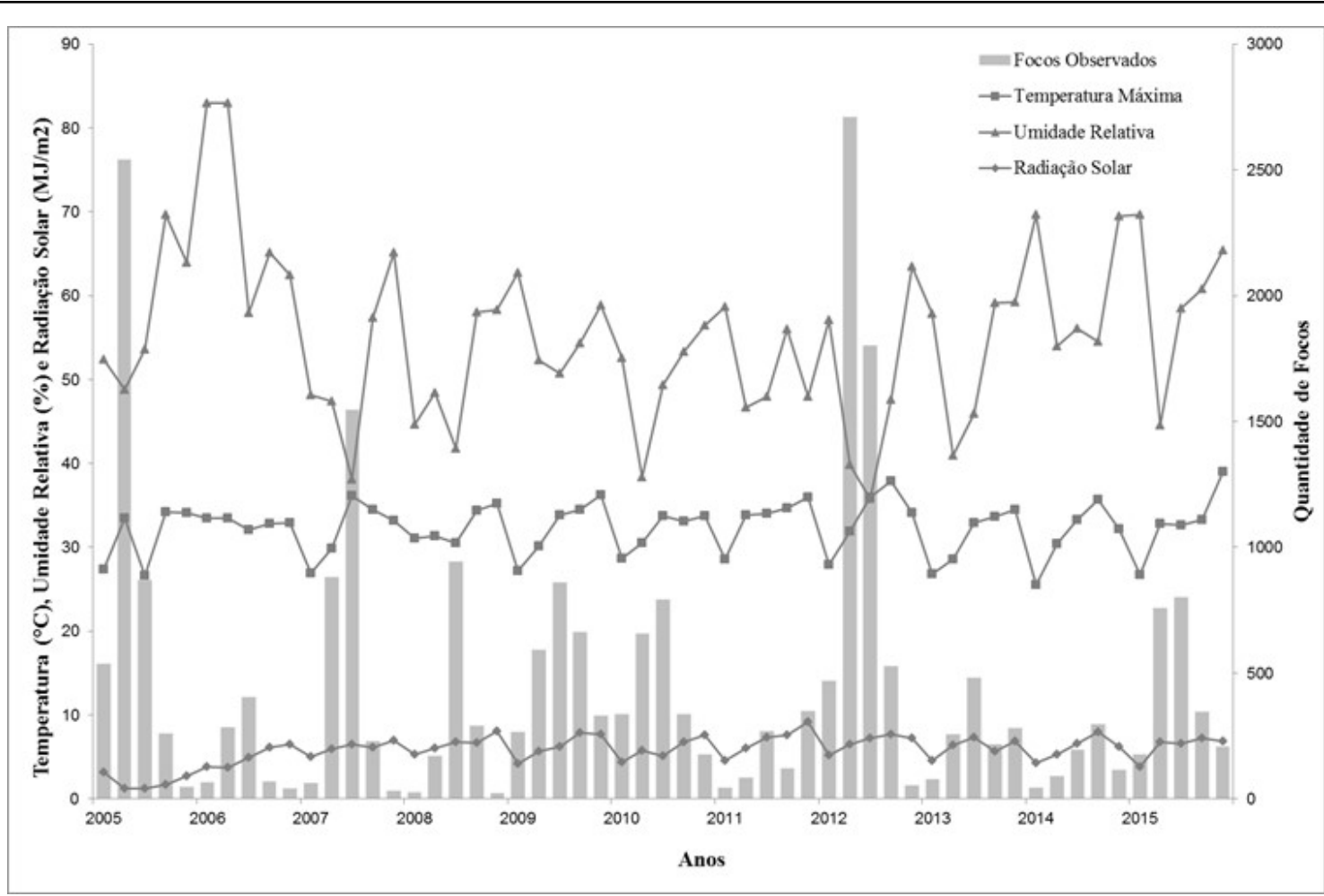

Figura 1. Variabilidade das médias mensais dos dados meteorológicos (Temperatura, Radiação e Umidade Relativa), relacionadas ao número total de focos para o período JASON da série temporal de 2005 a 2015, no município de Corumbá (MS).

Analisando o comportamento das variáveis meteorológicas com relação ao número de focos, verifica-se que, em geral, os maiores valores do número de focos ocorreram para os menores valores da umidade relativa, mostrando a correlação inversa entre essas duas variáveis como pode ser observado para o mês de setembro dos anos de 2007 e 2012. A baixa umidade relativa do ar influencia diretamente na vegetação, tornando-a mais seca, o que torna propício o aumento de material combustível e, consequentemente, a susceptibilidade ao processo de combustão. Os picos dos focos ocorreram para os meses de agosto e setembro do período de estiagem, com valor médio em torno de 450 focos.

A temperatura máxima média foi de $32,3^{\circ} \mathrm{C}$ e pode-se observar que para as temperaturas mais elevadas o número de focos é maior como, por exemplo, agosto de 2005, setembro de 2007, agosto e setembro de 2012 e novembro de 2014. A temperatura apresentou correlação com o número de focos, mas precisa estar associada ao fator da baixa umidade relativa para que possa ser significativa dentro dos modelos, pois se a temperatura for elevada e a umidade relativa do ar também, observa-se então uma diminuição do número de focos como ocorreram nos meses de agosto de 2006 e outubro de 2013.

A radiação solar apresentou valor máximo de 9,2 MJ m-2, entretanto, alta taxa de radiação não significa que o número de focos será máximo, pois, a sua incidência é diretamente afetada pela presença de nuvens. Quando ocorre baixa umidade e alta temperatura as curvas se apresentam em fase (ou seja, os pontos de máximo estão alinhados), porém, analisadas isoladamente, ocorrem defasagens entre as curvas de focos e radiação. Pode-se observar que setembro de 2007 as curvas estão em fase, e em novembro de 2014 observa-se que a radiação e a temperatura são elevadas, porém, em decorrência da alta umidade relativa do ar a ocorrência de focos decresce, representando essa defasagem da análise isoladamente.

Destacam-se os anos de 2005 e 2012 que apresentaram os maiores valores de incidências de focos da série, isso se deve ao acúmulo de material combustível das plantas dos anos precedentes (2004 e 2011, respectivamente), em que ocorreram grandes enchentes no

\section{IPABH}


município, tornando a vegetação mais fraca e propícia à queima, quando submetida às condições climáticas das altas temperaturas e radiação solar e baixa umidade relativa do ar nos anos seguintes, conforme pode ser observado na Figura 1.

Os anos de 2006, 2011 e 2014 apresentaram uma média de focos em torno de 170 registros, quantitativo inferior decorrente da alta umidade relativa apresentando a média acima de $60 \%$.

É apresentado, na Equação 1, o modelo RLM de previsão de focos de queimadas para o município de Corumbá (MS).

$\log (\widehat{N})=1,335-0,032 \times R-0,028 \times U+0,050 \times T$

Onde $\widehat{N}$ é o valor estimado para o número de focos; R é a radiação solar acumulada; U é a umidade relativa; e T é a temperatura máxima.

A estatística descritiva do modelo de regressão aplicado, com variável dependente $\log (\widehat{N})$ e as variáveis preditoras Radiação Solar, Umidade Relativa e Temperatura, resultou no valor de $\mathrm{R}^{2}=0,411$ e indica que o grau de ajustamento das variáveis preditivas foi de $41 \%$ com $\mathrm{p}<0,001$, explicando a variabilidade de saída pelos dados das variáveis meteorológicas preditoras. O erro percentual absoluto médio (MAPE) foi de 33,9\%, cometido entre os valores do logaritmo decimal do número de focos para os valores estimados pela Equação 1. Ao se aplicar o teste de Stein no valor de $\mathrm{R}^{2}$ foi possível verificar que o resultado foi próximo do valor de ajuste resultando em 0,410, o que significa que o modelo pode ser generalizado para diferentes amostras, ocorrendo validação cruzada do modelo.

A estatística F resultou no valor 390,034, que significa que novas variáveis preditoras inseridas no modelo pela configuração do software IBM-SPSS, no modo "stepwise, foi satisfatória para a previsão do número de focos, entretanto a hipótese de independência dos erros analisada pelo teste de Durbin-Watson, foi parcialmente satisfatória, resultando em 0,917.

Com essas análises, pode-se verificar que a técnica de RLM não é o modelo mais adequado para a previsão do número de focos (quando se utiliza como variáveis preditoras somente os dados meteorológicos), pois, não se apresentou estritamente linear e deve-se levar em consideração ainda a possibilidade de inserir outras variáveis que possam contribuir para aumentar o desempenho desse método, como, por exemplo, uma variável associada à ação antrópica, da qual identifica as queimadas de acordo com a ação humana e classifica os focos provenientes de ação natural ou não (White e White, 2016).

Na aplicação da técnica ARIMA de Box-Jenkins, utilizando os dados meteorológicos como variáveis preditoras, houve uma otimização da previsão com o modelo $\operatorname{ARIMA~}(4,0,10)$, ou seja, com quatro termos autorregressivos e dez termos da média móvel. Não foi necessária a diferenciação, ou seja, $d=0$, por se tratar de dados estacionários. O número de ordens autorregressivas foi $\mathrm{p}=4$, sendo necessários quatro períodos de tempo da série no passado para prever o valor atual. $\mathrm{O}$ valor de $\mathrm{q}=10$ especifica que os desvios dos valores médios das séries de cada um dos dez últimos períodos de tempo, são considerados, ao prever os valores atuais da série.

A análise do critério de informações bayesianas aplicadas para comparar os modelos, demonstrou que o modelo ARIMA $(4,0,10)$ é o melhor modelo previsor associado à significância $\mathrm{p}<0,05$. O ajuste do modelo passa a ser de $66,5 \%$, com um erro percentual absoluto médio (MAPE) de 31,6\%, melhorando consideravelmente o desempenho geral do modelo ARIMA ao se comparar à modelagem RLM.

Os gráficos comparativos dos valores dos focos observados e previstos pelas técnicas de previsão RLM e ARIMA estão representados na Figura 2 (a) e (b), respectivamente. 

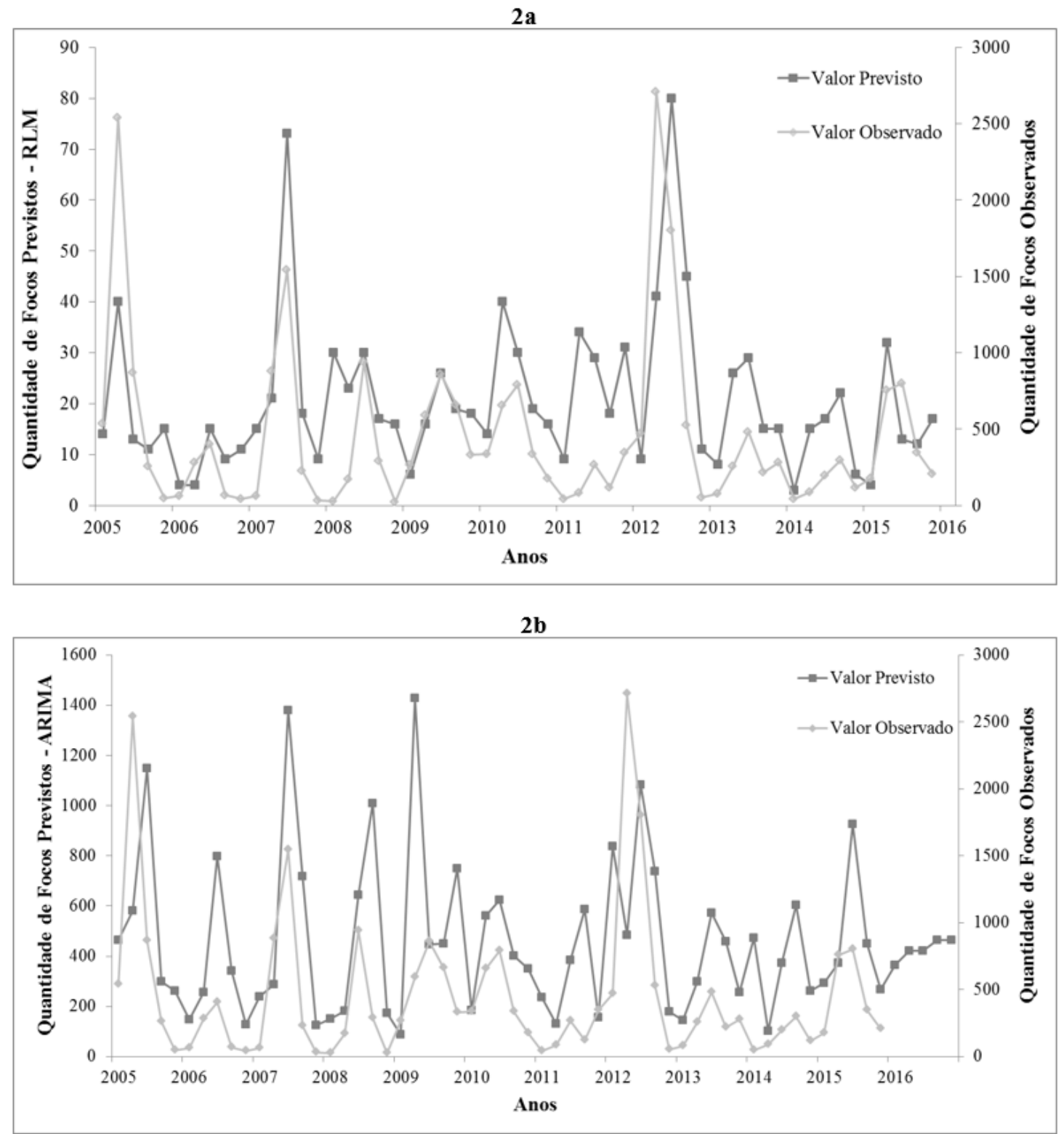

Figura 2. Valores observados e previstos dos focos obtidos nas modelagens de RLM (Figura 2a) e ARIMA (Figura 2b), no período JASON para a série histórica de 2005 a 2015 no município de Corumbá (MS).

De acordo com a Figura 2a para o modelo RLM, é possível constatar que os gráficos dos focos previstos e dos focos observados estão em fase com picos de máximo e mínimo coincidentes e o crescimento e decrescimento com as mesmas características ao longo de toda a curva, em diferentes escalas por terem sido aplicados de forma parametrizada na regressão com a utilização do logaritmo decimal, cabe salientar, que para obter os dados previstos, aplicase o antilogaritmo no resultado da equação de modelagem. No entanto, o viés da previsão foi subestimar os valores dos focos, acertando em menos de $50 \%$ os dados de saída. A partir da Figura 2b para o modelo ARIMA é possível observar que as curvas dos valores previstos e dos valores estimados estão em fase e apresentam as condições de tonicidade coincidentes e os picos de máximo e mínimo com o mesmo delineamento, sendo possível de realizar a previsão com cinco passos posteriores com essa mesma precisão, e apesar de também subestimar os dados de saída, os acertos foram superiores a $50 \%$ dos casos.

Os gráficos dos resíduos dos dois modelos estão representados na Figura 3, sendo, para o RLM (Figura 3a) e para o ARIMA (Figura 3b), respectivamente.

\section{IPABH}



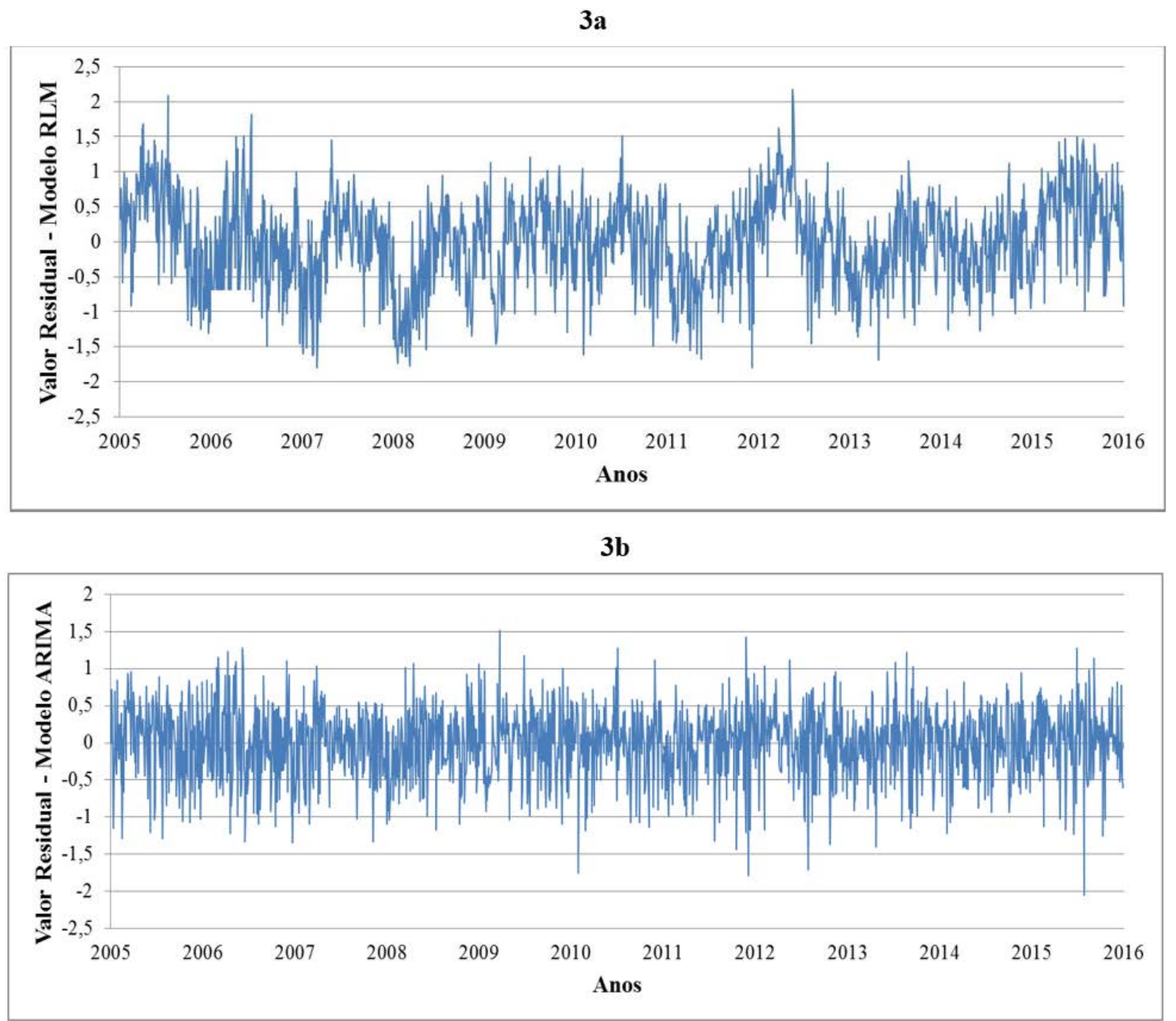

Figura 3. Valores residuais nas modelagens de RLM (Figura 2a) e ARIMA (Figura 2b), no período JASON para a série histórica de 2005 a 2015 do município de Corumbá (MS).

Considerando a análise de resíduos, pode-se enfatizar que o modelo ARIMA pode ser utilizado para fazer inferências além da amostra de dados utilizadas, entretanto o modelo RLM (Figura 3a) para a previsão dos focos a partir de dados preditores estritamente meteorológicos, não se apresentou uma técnica adequada para a previsão, o que evidencia não se tratar de um modelo de características estritamente lineares, e os resíduos oscilam consideravelmente em torno de zero. Já a análise do modelo ARIMA explica a variância das variáveis meteorológicas para a previsão dos focos no que tange em quão propício estão de ocorrer. Para melhorar a previsão de quantos focos poderão ocorrer é necessário inserir variáveis que especifiquem as causas da ocorrência de cada foco (naturais ou antrópicas), ou até mesmo variáveis da área agrícola e pecuária.

Na análise dos resíduos na modelagem ARIMA (Figura 3b), o teste de Durbin-Watson $(\mathrm{D}=1,97)$ mostrou que a hipótese de interdependência dos erros é satisfeita, pois se apresenta próxima do valor esperado (2) e o desempenho geral da técnica foi de $68,4 \%$ com p $<0,002$, sendo capaz de fazer a previsão cinco passos adiante com essa mesma precisão.

A partir da análise do gráfico de resíduos, pode-se ainda constatar que o modelo ARIMA é mais ajustado, pois apresenta valores mais próximos de zero e com menor coeficiente de variação. Os estimadores não são enviesados e o modelo ARIMA apresentou resultados significativamente melhores para prever a saída de dados do número de focos a partir das variáveis meteorológicas.

As modelagens de RLM e ARIMA foram aplicadas para a previsão do número de focos a 
partir de dados preditores meteorológicos, e foi possível observar que há a necessidade de inserir outras variáveis para se utilizar esse tipo de técnica de previsão, no intuito de melhorar a saída de dados e consequentemente diminuir os valores residuais.

\section{CONCLUSÕES}

Foi possível verificar a correlação dos fatores meteorológicos com o número de focos, que demonstraram a partir dos resultados da correlação de Pearson e da análise gráfica que essas variáveis guardam uma importante relação com as condições da vegetação do Pantanal de Corumbá-MS e afetam diretamente a ocorrência de focos de queimadas e incêndios, onde se destacaram a radiação solar, a umidade relativa e a temperatura.

Foram aplicadas as técnicas de análises multivariadas de dados utilizando as modelagens RLM e ARIMA, em que o modelo RLM explicou $41 \%$ da variância do número de focos, mostrando-se uma técnica não eficiente para a previsão, no que tange as variáveis meteorológicas como preditoras, demonstrando a necessidade de inserir outras variáveis relacionadas à ação antrópica, tais como as atividades de agropecuária.

Já, o modelo ARIMA $(4,0,10)$ apresentou resultados mais satisfatórios em relação à modelagem RLM, possibilitando explicar a variância do número de focos em 66,5\%, o que demonstra ser a técnica mais adequada para a previsão quando comparada com a técnica RLM. No entanto, espera-se que o desempenho dessa técnica melhore ao se realizar a inserção de outras variáveis. Outras modelagens podem ser testadas para a previsão dos focos a partir das variáveis estritamente meteorológicas e fica como sugestão para trabalhos futuros.

\section{REFERÊNCIAS}

ALMEIDA, C. T.; OLIVEIRA JÚNIOR, J. F.; DELGADO, R. C.; CUBO, P.; RAMOS, M. C. Spatiotemporal rainfall and temperature trends throughout the Brazilian Legal Amazon, 1973-2013. International Journal of Climatology, v. 37, n. 4, p. 2013-2026, 2016. https://doi.org/10.1002/joc.4831

ARTAXO, P.; OLIVEIRA, P, H.; LARA, L. L.; PAULIQUEVIS, T. M.; RIZZO, L. V.; JUNIOR, C. P. Efeitos climáticos de partículas de aerossóis biogênicos e emitidos em queimadas na Amazônia. Revista Brasileira de Meteorologia, v. 21, n. 3a, p. 168-189, 2006.

BRASIL. Ministério das Minas e Energia. RadamBrasil. Folha SE. 21. Corumbá e Folha SE. 20. Geologia, Pedologia, Geomorfologia, Vegetação e Uso e Ocupação da Terra. Rio de Janeiro,1982. 448 p.

BRITO, T. T.; OLIVEIRA JÚNIOR, J. F.; LYRA, G. B.; GOIS, G.; ZERI, M. Multivariate analysis applied to monthly rainfall over Rio de Janeiro state, Brazil. Meteorology and Atmospheric Physics, v. 129, n. 5, p. 469-478, 2016.

CAÚLA, R. H.; OLIVEIRA JÚNIOR, J. F.; GOIS, G.; DELGADO, R. C.; PIMENTEL, L. C. G.; TEODORO, P. E. Nonparametric statistics applied to fire foci obtained by meteorological satellites and their relationship to the MCD12Q1 product in the state of Rio de Janeiro, Southeast - Brazil. Land Degradation \& Development, v. 28, n. 3, p. 1056-1067, 2016. https://doi.org/10.1002/ldr.2574

CLEMENTE, S. S.; OLIVEIRA JÚNIOR, J. F.; LOUZADA, M. A. P. Focos de calor na Mata Atlântica do Estado do Rio de Janeiro. Revista Brasileira de Meteorologia, v. 32, n. 4, p. 669-677, 2017. 
DEPPE, F.; PAULA, E. V.; MENEGHETTE, C. R.; VOSGERAU, J. Comparação de índice de risco de incêndio florestal com focos de calor no Estado do Paraná. Floresta, v. 34, n. 2, p. 119-126, 2004. http://dx.doi.org/10.5380/rf.v34i2.2382

FREITAS, S. R.; LONGO, K. M.; SILVA DIAS, M. A. F. S.; SILVA DIAS, P. L. S. Emissões de Queimadas em ecossistemas da América do Sul. Estudos Avançados, v. 19, n. 53, p. 167-185, 2005. http://dx.doi.org/10.1590/S0103-40142005000100011

GONÇALVES, A. B.; VIEIRA, A. Grandes incêndios florestais, erosão, degradação e medidas de recuperação dos solos. Minho: Universidade do Minho, 2013. 299 p.

HAIR JR., J.; ANDERSON, R.; TATHAM, R.; BLACK, W. Análise multivariada de dados. Porto Alegre: Bookman, 2005. 596 p.
INSTITUTO BRASILEIRO DE GEOGRAFIA E ESTATÍSTICA - IBGE. Cidades Mato Grosso do Sul - Corumbá MS. Disponível em: https://cidades.ibge.gov.br/brasil/ms/corumba. Acesso em: 08 fev. 2016.

MAGI, B. I.; RABIN, S.; SHEVLIAKOVA, E.; PACALA, S. Separating agricultural and nonagricultural fire seasonality at regional scales. Biogeosciences, v. 9, n. 8, p. 3003-3012, 2012. https://doi.org/10.5194/bg-9-3003-2012

MORETTIN, P. A.; TOLOI, C. M. C. Análise de séries temporais. São Paulo: Edgard Blüncher, 2006. 531 p.

MORRISON, J. H. Caring for country: indigenous people managing country using fire, with particular emphasis on Northern Australia. In: INTERNATIONAL WILDLAND FIRE CONFERENCE, 9., 2014, Sydney. Abstract and Papers... Sydney: ISDR, 2014.

NOGUEIRA, J. S.; SANTOS, A. C. A. Análise do material particulado no Pantanal MatoGrossense. Revista Brasileira de Meteorologia, v. 30, n. 3, p. 254-264, 2015.

NUNES, M. T. O.; SOUSA, G. M.; TOMZHINSKI, G. W.; OLIVEIRA-JÚNIOR, J. F.; FERNANDES, M. C. Factors Influencing on Susceptibility Forestry Fire in Itatiaia National Park. Anuário do Instituto de Geociências, v. 38, n. 1, p. 54-62, 2015.

PEREIRA, A. A.; PEREIRA, J. A. A.; MORELLI, F.; BARROS, D. A.; ACERBI JR., F. W.; SCOLFORO, J. R. S. Validação de focos de calor utilizados no monitoramento orbital de queimadas por meio de imagens TM. Cerne, v. 18, p. 335-343, 2012.

SANTOS, A. C. A.; NOGUEIRA, J. S. Análise do material particulado no Pantanal MatoGrossense. Revista Brasileira de Meteorologia, v. 30, n. 3, p. 254-264, 2015.

SILVA, R. A. Incêndio Florestal. In: TOCCHETTO, D. Perícia ambiental criminal. Campinas: Millennium, 2014. p. 351 - 406.

SOARES, R. V.; BATISTA, A. C.; NUNES, J. R. S. Incêndios florestais no Brasil: o estado da arte. Curitiba: Produção Independente, 2009. 246 p.

TORRES, F. T. P.; RIBEIRO, G. A.; MARTINS, S. V.; LIMA, G. S. Correlações entre os elementos meteorológicos e as ocorrências de incêndios florestais na área urbana de Juiz de Fora, MG. Revista Árvore, v. 35, n. 1, p. 143-150, 2011. http://dx.doi.org/10.1590/S0100-67622011000100017 
TREJO, D. A. R. Fire Regimes, Fire Ecology, and Fire Management in Mexico. Journal of the Human Environment, v. 37, n. 7, p. 548-556, 2008. https://doi.org/10.1579/0044-744737.7.548

VIGANÓ, H. H. G.; SOUZA, C. C.; CRISTALDO, M. F.; JESUS, L. Redes Neurais Artificiais na Previsão de Queimadas e Incêndios no Pantanal. Revista Brasileira de Geografia Física, v. 10, n. 5, p. 1355-1367, 2017. http://dx.doi.org/10.26848/rbgf.v.10.p1355-1367

WHITE, B. L. A.; WHITE, L. A. S. Queimadas controladas e incêndios florestais no Estado de Sergipe, Brasil, entre 1999 e 2015. Floresta, v. 46, n. 4, p. 561-570, 2016. http://dx.doi.org/10.5380/rf.v46i4.47036 